\title{
Passive scalar mixing in a planar shear layer with laminar and turbulent inlet conditions
}

\author{
L. M. Pickett and J. B. Ghandhi ${ }^{\mathrm{a})}$ \\ Engine Research Center, Department of Mechanical Engineering, University of Wisconsin-Madison, \\ Madison, Wisconsin 53706
}

(Received 13 February 2001; accepted 29 November 2001)

\begin{abstract}
The effect of inlet conditions on the mixing of a passive scalar was investigated in a planar shear layer with inlet boundary layers that were laminar, tripped and naturally turbulent-transitional. Planar laser-induced fluorescence measurements of acetone were used to directly evaluate the shear layer structure, and were processed to determine probability density functions (PDFs) of the mixture fraction. The results agree well with previous studies in aqueous and gaseous systems for laminar inlet conditions. Large-scale structures of a nearly homogeneous composition were found, and the structures spanned the mixing layer width giving rise to a nonmarching style PDF. A high-speed boundary layer that differed from the laminar state (produced by tripping a laminar boundary layer, from the naturally turbulent-transitional state at high flow rates, or by tripping the turbulenttransitional condition) gave rise to a hybrid-style PDF that was markedly different from either the laminar nonmarching case, or the marching shape that has been found by other investigators. The hybrid PDF shape had a marching character on the high-speed side of the mixing layer, and a distributed nature not favoring any specific composition on the low-speed side of the mixing layer. Tripping the low-speed boundary layer produced no change in the hybrid PDF shape, confirming that the difference observed between the high- and low-speed sides was the result of the shear layer development with turbulent inlet conditions, not an inlet condition effect. In addition, with turbulent and transitional inlet conditions all turbulent passive scalar profiles were found to be self-similar and the velocity power spectra displayed a $-5 / 3$ slope indicating well-developed turbulent conditions prevailed at a relatively low $\left(10^{4}\right)$ Reynolds number. Secondary structures were observed in images with turbulent-transitional inlet conditions, but not with tripped inlet conditions. (C) 2002 American Institute of Physics. [DOI: 10.1063/1.1445421]
\end{abstract}

\section{INTRODUCTION}

In planar mixing layer experiments the freestream velocity and density of the two streams are fixed, allowing study of the mixing layer with carefully controlled boundary conditions. Despite the simplification, experiments have shown a high degree of sensitivity to the state of the inlet boundary conditions even for measurements performed at highReynolds number, far downstream of the splitter plate tip. The mixing layer growth rate, for instance, has been shown to be significantly different for either laminar or turbulent boundary layer inlet conditions. Half-jet mixing layers, where the low-speed freestream velocity is zero, have shown a larger growth rate when the boundary layer is turbulent. ${ }^{1,2}$ In contrast, two-stream mixing layers with a nonzero velocity in each freestream have shown a reduced growth rate when the boundary layer is turbulent. ${ }^{3-5}$

For laminar inlet conditions numerous criteria have been suggested as being necessary before a self-similar flow is obtained (where the transverse coordinate is normalized by the local layer width). In a half-jet, Bradshaw ${ }^{6}$ found that self-similarity was not obtained until a streamwise position,

\footnotetext{
a) Author to whom correspondence should be addressed. Telephone: (608) 263-1684; Fax: (608) 263-9870. Electronic mail: ghandhi@engr.wisc.edu
}

$x=1000 \theta_{0}$, where $\theta_{0}$ is the high-speed boundary layer momentum thickness, while other researchers have found selfsimilarity to be achieved both earlier ${ }^{7}$ and later. ${ }^{5}$ The minimum downstream distance for self-similarity has also been expressed in terms of the number of large-scale structure interactions. ${ }^{89}$ Huang and $\mathrm{Ho}^{9}$ showed that the exponent of power spectra reached the asymptotic $-5 / 3$ value at a normalized streamwise coordinate, $R x / \lambda_{0} \approx 8$, where $R=(1$ $-r) /(1+r), r$ is the velocity ratio $U_{2} / U_{1}$, and $\lambda_{0}$ is the wavelength of the initial structure. At the position $R x / \lambda_{0}$ $\approx 8$, approximately two structure pairings had occurred over a wide range of velocity ratios indicating a correlation between the large-scale behavior and self-similarity of the mixing layer. In a two-stream mixing layer, Bell and Mehta ${ }^{5}$ found agreement between the far-field turbulent profiles for laminar and turbulent inlet conditions, but self-similarity occurred much earlier with turbulent inlet conditions.

Although the velocity data with either laminar or turbulent inlet conditions suggests convergence to a final selfsimilar state, passive scalar measurements have shown less agreement. Typically viewed in terms of the probability density function (PDF), either nonmarching, ${ }^{10,11}$ where a preferred composition extends across the layer, or marching, ${ }^{12}$ where the most probable composition varies linearly across the layer, style PDFs have been observed in planar mixing 
layers at similar Reynolds number, $\operatorname{Re}_{\delta} \equiv \Delta U \delta \nu^{-1}$, where $\Delta U$ is the velocity difference of the freestreams and $\delta$ is the mixing layer thickness based on the $1 \%$ value of the freestream mixture composition. In a liquid mixing layer with laminar inlet conditions, Karasso and Mungal ${ }^{13}$ found that the PDF shape depended solely on the value of the pairing parameter, $R x / \lambda_{0}$. For values of the pairing parameter less than a critical value of 22 the PDFs were nonmarching, and for values in excess of this critical value the PDFs were marching. Although the PDFs presented at high values of the pairing parameter were marching, the measurements were under-resolved and the authors suggest that the fully developed state would be characterized by a tilted PDF. For some instances tripping the high-speed boundary layer was found to cause a change of a nonmarching PDF to a marching PDF, but this was not found to be true in all cases.

Passive scalar measuring techniques tend to overestimate the extent of molecular mixing due to the finite size of the measurement volume. When the mixing scale is smaller than the sampling volume, unmixed but stirred fluid appears as mixed and passive scalar gradients are smoothed. The resolution of the experiment can be expressed in terms of the local mass diffusion scale, $\lambda_{D}$, which represents the smallest physical length scale for mixing, as $\lambda_{D} / \delta$ $=C_{b} \operatorname{Re}_{\delta}^{-3 / 4} S c^{-1 / 2}$ where a value of $C_{b}=11$ has been suggested by Buch and Dahm. ${ }^{14}$ In comparison to this parameter, the experimental resolution, $L$, in liquid mixing layers has been relatively poor $\left(L / \lambda_{D}>71^{13}\right)$ due to the high Schmidt numbers. The data acquired in gaseous mixing layer studies has not resolved the mixing scale, but is much better than the liquid studies (e.g., Batt, ${ }^{12} L / \lambda_{D} \approx 10$ ). In liquid mixing layers, it has been suggested that the observed shift in PDF style from nonmarching to marching with turbulent inlet conditions or higher $\operatorname{Re}_{\delta}$ is not physical but is due to measurement resolution problems. ${ }^{11,13}$ Thus, the effect of turbulent inlet conditions on scalar mixing is somewhat uncertain.

Recently, product measurements were made in a gaseous reacting mixing layer with fast chemistry by Slessor et al. ${ }^{15}$ By performing separate "flip" experiments where the lean reactant was placed in either the low- or high-speed stream, the product measurements were used as a resolution-free approximation of the amount of molecularly mixed fluid. Experiments with either laminar or turbulent inlet conditions were performed at $\operatorname{Re}_{\delta}=2 \times 10^{5}, x / \theta_{0}=3300$ and $R x / \lambda_{0}$ $\approx 47$, thus all previous criteria suggested for self-similar flow were satisfied. The product profiles with laminar and turbulent boundary layer inlet conditions showed strong differences. With laminar inlet conditions, the product profile in both flip experiments was symmetric indicating a nonmarching style PDF while with turbulent inlet conditions the product profile favored the lean reactant, more representative of a marching style PDF. The mixed fluid profile was, therefore, not a unique function of local conditions, but remained sensitive to the state of the inlet conditions even in conditions considered to be fully developed. However, the shape of the PDF was only inferred from the results, not directly measured.
The effect of initial conditions on the PDF shape has also been investigated through direct numerical simulation (DNS) of temporally developing mixing layers. A recent study by Cortesi et al. ${ }^{16}$ showed that when the domain was initialized with two-dimensional perturbations to the mean profile a nonmarching PDF was obtained at the end of the simulation, but marching or tilted PDFs resulted in the final stage of the simulation with three-dimensional perturbations. A three-dimensional initial condition with a deterministic forcing that promoted rapid development of streamwise rib vortices produced a marching PDF, but a randomized threedimensional perturbation produced a tilted PDF. Using turbulent boundary layer data obtained from a previous DNS study as an initial condition, Rogers and Moser $^{17}$ found a marching PDF shape. Ansari ${ }^{18}$ studied mixing layers initialized with both low and high-gradient laminar boundary layers and found final stage PDFs that were nonmarching for the low-gradient laminar boundary layer but marching for the high-gradient laminar boundary layer. Differences in inlet conditions are, therefore, shown to exert a dominant effect on the final PDF shape in these DNS studies, although experimental verification is still limited.

The research described here represents passive scalar measurements of a gaseous mixing layer with both laminar and turbulent inlet conditions performed using planar laserinduced fluorescence (PLIF) of a tracer molecule. The passive scalar technique is used to directly obtain the PDF shape. Although the resolution of the passive scalar technique has been questioned, especially for liquid mixing layer studies, a separate technique ${ }^{19}$ recently showed minimal subresolution stirring in a gaseous system, and previous measurements with this technique in our own facility showed adequate resolution of mixing scales. ${ }^{20}$ The objective of this research is to study the influence of inlet conditions on the mixing behavior of the system as provided by the passive scalar structure and PDF shape. The detailed behavior will be emphasized as compared to the global changes effected by inlet conditions as has been previously described in the literature. Understanding these effects in a nonreacting mixing layer is the first step in being able to predict behavior in practical devices for which well-condition inlet streams are not expected.

\section{EXPERIMENTAL APPROACH}

\section{A. Shear layer facility}

A steady flow apparatus, fed by the building compressed air supply, was used for the reported experiments. Both air streams were metered by choked orifice plates comprised of $\sim 80$ small diameter holes. The two airstreams separately entered an outer pressure containment vessel and passed through a series of flow conditioning elements, consisting of perforated plates, tube bundles, and mesh screens that reduce the turbulence level and isolate upstream acoustical modes from the shear layer. After the final series of screens, a symmetrical nozzle and a $3.8^{\circ}$ tapered splitter plate (nominal tip thickness is $0.1 \mathrm{~mm}$ ) were used to create the shear flow, see Fig. 1. In this geometry, the high-speed and low-speed streams pass through a 1.75:1 contraction ratio. The nozzle 


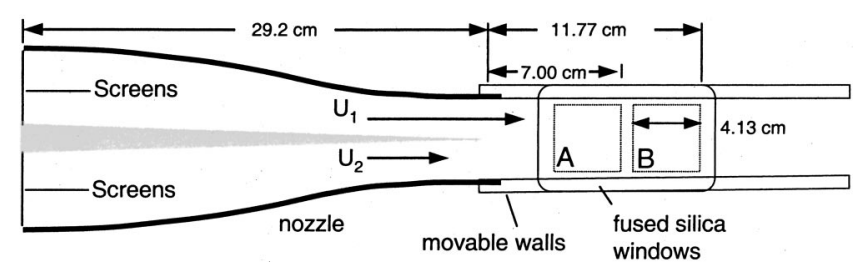

FIG. 1. Shear layer facility.

served to thin the boundary layer on the splitter plate and to produce a flat velocity profile at the exit plane. The test section was $5 \mathrm{~cm}$ in the cross-stream direction and $20 \mathrm{~cm}$ in the spanwise direction. The rectangular test section had two hinged walls that were used to adjust the streamwise pressure gradient in the flow. For all tests reported here, the walls were adjusted so that the streamwise pressure gradient was zero. Optical access is provided with fused silica windows at each quadrant of the test section. The entire flow conditioning run and test section is enclosed in the pressurized containment vessel which is designed for operation at pressures up to $6 \mathrm{~atm}$. At the top of the containment vessel, the gases are throttled to atmospheric pressure before entering the exhaust system. Further details of the facility can be found in Pickett. ${ }^{21}$ Extensive combusting tests with a high level of heat release have been performed in this facility, and the lack of any evidence of acoustical coupling with the heat release suggests that the facility is free from acoustical disturbances which could artificially excite the layer.

\section{B. Planar laser-induced fluorescence of acetone}

Planar laser-induced fluorescence (PLIF) was performed with acetone vapor seeded into one side of the mixing layer. The acetone was added to either the low- or high-speed fluid by a jet of atomizing droplets issuing from a small orifice located far upstream of the tip of the splitter plate. The seeding rate was known by controlling the liquid pressure above the orifice. The fluorescence signal is linearly proportional to the concentration of acetone ${ }^{22}$ thus scalar mixing can be visualized in the two-dimensional flowfield with excellent spatial and temporal resolution.

The fourth harmonic $(266 \mathrm{~nm})$ of a Nd:YAG laser was formed into a $650 \mu \mathrm{m}$ (maximum) sheet to excite the acetone fluorescence. Images $4.13 \times 4.13 \mathrm{~cm}$ in size were collected at two streamwise positions (labeled A and B in Fig. 1) extending to $11.77 \mathrm{~cm}$ downstream from the tip of the splitter plate. The fluorescence signal was collected with an Apogee AP7 cooled, back-illuminated, slow-scan CCD camera and binned to a $256 \times 256$ pixel array. A Nikkor $105 \mathrm{~mm}$ focal length, $f / 2.8$ lens was used as the collection optic and effectively filtered the ultraviolet (UV) light of the laser from the fluorescence signal. Each binned pixel corresponded to a measurement area at the laser sheet of $160 \times 160 \mu \mathrm{m}$. Each individual image was corrected for dark noise, background scattering, laser energy and profile variations, and absorption of the incident beam. The laser pulse energy and profile variations were corrected by using the uniformly seeded freestream as a reference. ${ }^{21}$ The signal to noise ratio was confirmed to be governed by the photonic shot noise, and was 34:1 in the high-speed freestream for the binned pixels.

The spatial resolution of the images was limited by the thickness of the laser sheet and not the pixel size due to the three-dimensional nature of the flow field. Using this length scale and the diffusion length scale defined above, the relative resolution $L / \lambda_{D}$ was found to range from 1.2 to 3.2 for the conditions investigated. Therefore, it is expected that there can be some sub-pixel stirring, but it is not expected to strongly bias the results. Under similar conditions, King et al. ${ }^{19}$ found minimal sub-resolution mixing.

One hundred temporally uncorrelated, single-shot images were obtained at each test condition for statistical analysis. The data over 20 adjacent columns were compiled to compute the PDF shape since the thickness of the mixing layer changes by less than $2 \%$ over this distance. ${ }^{20}$ The shape of the PDF was unchanged compared to using single pixelwidth column data, and the effect of using multiple columns was merely to minimize the effect of random noise.

The results are presented as the mixture fraction of highspeed fluid, $\xi$, which is linearly proportional to the acetone mole fraction or $\xi=x_{a} / x_{1}$ where $x_{a}$ is the measured mole fraction of acetone and $x_{1}$ is the mole fraction of acetone in the high-speed stream. The PDF of mixture fraction of highspeed fluid, $p(\xi, x, y)$, at a point (pixel) in the mixing layer was assembled by binning the mixture fraction data into 25 equally spaced bins ranging from 0 to 1 . The PDF was normalized such that

$$
\int_{0}^{1} p(\xi, x, y) d \xi=1
$$

The mean and root mean square (rms) mixture fraction values can be directly determined from the PDF.

The mixed fluid probability, $P_{m}$, is defined as

$$
P_{m}(x, y)=\int_{\varepsilon}^{1-\varepsilon} p(\xi, x, y) d \xi,
$$

where $\varepsilon$ is chosen to exclude pure high- or low-speed fluid. In order to discriminate against noise a value of $\varepsilon=0.12$ was chosen, thus $P_{m}$ is the probability that $0.12<\xi<0.88$ fluid will be measured.

The average mixed-fluid mixture fraction is defined as

$$
\xi_{m}(x, y)=\frac{\int_{\varepsilon}^{1-\varepsilon} \xi p(\xi, x, y) d \xi}{P_{m}(x, y)},
$$

which describes the preferred mixed-fluid composition at a point in the layer. The total mixed-fluid mixture fraction for a cross section taken across the layer is defined as

$$
\xi_{M}(x)=\frac{\int_{-\infty}^{\infty} P_{m}(y) \xi_{m}(y) d y}{\int_{-\infty}^{\infty} P_{m}(y) d y} .
$$

\section{Test conditions}

The effect of inlet conditions was examined by performing experiments with laminar, naturally turbulent and tripped high-speed boundary layers. The experimental test conditions are shown in Table I. In all cases but one (case 8) the low-speed boundary layer was laminar. For laminar inlet conditions, the momentum thickness of the high-speed boundary layer, $\theta_{0}$, was calculated from the geometry of the 
TABLE I. Operating conditions for passive scalar tests.

\begin{tabular}{|c|c|c|c|c|c|c|c|c|c|}
\hline Case & $\begin{array}{c}\text { High-speed } \\
\text { boundary layer }\end{array}$ & $\begin{array}{c}U_{1} \\
(\mathrm{~m} / \mathrm{s})\end{array}$ & $r$ & $\begin{array}{c}P \\
(\mathrm{~atm})\end{array}$ & $\begin{array}{c}\theta_{0} \\
(\mathrm{~mm})\end{array}$ & $\begin{array}{c}\delta(\mathrm{cm}) \\
x=11 \mathrm{~cm}\end{array}$ & $\operatorname{Re}_{\delta}$ & $\begin{array}{c}\xi_{M} \\
x=11 \mathrm{~cm}\end{array}$ & PDF type \\
\hline 1 & Tripped (weakly) & 2 & 0.4 & 2 & $0.66^{\mathrm{a}}$ & 2.15 & 3300 & 0.57 & Hybrid \\
\hline 2 & Laminar & 3 & 0.4 & 2 & $\begin{array}{l}0.54^{\mathrm{a}} \\
0.47^{\mathrm{b}}\end{array}$ & 2.41 & 5500 & 0.53 & Nonmarching \\
\hline 3 & Tripped & 3 & 0.4 & 2 & $\cdots$ & 2.17 & 5000 & 0.55 & Hybrid \\
\hline 4 & Laminar & 4 & 0.3 & 2 & $0.47^{\mathrm{a}}$ & 2.71 & 9600 & 0.52 & Nonmarching \\
\hline 5 & Turbulent-transitional & 4 & 0.4 & 4 & $0.36^{\mathrm{b}}$ & 1.93 & 11800 & 0.55 & Hybrid \\
\hline 6 & Tripped-HS & 4 & 0.4 & 4 & $\ldots$ & 1.82 & 10500 & 0.55 & Hybrid \\
\hline 8 & Tripped-LS & 4 & 0.4 & 4 & $\cdots$ & 2.21 & 13500 & 0.55 & Hybrid \\
\hline 7 & Turbulent-transitional & 5 & 0.4 & 4 & $\cdots$ & 1.89 & 14400 & $\cdots$ & $\cdots$ \\
\hline
\end{tabular}

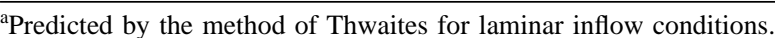

${ }^{\mathrm{b}}$ Measured by hot-wire anemometry.

contracting section using the method of Thwaites, or directly measured with hot-wire anemometry, with the latter providing slightly lower values. Tripping was achieved by installing a $2.36 \mathrm{~mm}$ diameter rod on one side of the splitter plate $153 \mathrm{~mm}$ upstream of the splitter plate tip. The size and position of the rod were calculated for the conditions of case 3 based on three criteria consistent with previous experiments using trip wires: $d_{\text {trip }} / \theta_{0}>5, \quad \operatorname{Re}_{\text {trip }}>850$, and $x_{\text {trip }} / \theta_{0}$ $=-300^{3,4,15}$ where $\operatorname{Re}_{\text {trip }} \equiv U_{1} d_{\text {trip }} \nu^{-1}$, and $\theta_{0}$ is the laminar momentum thickness at the splitter plate tip in the absence of the trip. A weakly tripped $\left(d_{\text {trip }} / \theta_{0}=3.5, \mathrm{Re}_{\text {trip }}=630\right.$, and $\left.x_{\text {trip }} / \theta_{0}=-230\right)$ condition, case 1 , was also tested. Finally, the effect of a low-speed side trip was investigated in case 8 .

Velocity measurements were performed with hot-wire anemometry ${ }^{21}$ under atmospheric pressure conditions to investigate the nature of the high-speed boundary layer. Due to system constraints, the measurements were taken at a streamwise position $1 \mathrm{~mm}$ past the splitter plate tip. The experiments were performed with the low-speed stream not flowing $\left(U_{2}=0\right)$ and with the high-speed velocity chosen to match the plate Reynolds numbers, $\operatorname{Re}_{x} \equiv U_{1} x / \nu$ where $x$ is the linear distance along the splitter plate, as cases $2\left(\operatorname{Re}_{x}\right.$ $\left.=1.1 \times 10^{5}\right)$ and $5\left(\operatorname{Re}_{x}=2.9 \times 10^{5}\right)$. The mean velocity profiles are shown in Fig. 2(a), where the Blasius profile has been fit to the data for $x=15 \mathrm{~cm}$ instead of the actual distance $(x=29 \mathrm{~cm})$ to account for the favorable pressure gradient due to the nozzle. The average profile at $\mathrm{Re}_{x}=1.1$ $\times 10^{5}$ closely matches the laminar profile, but deviations from a laminar profile are found for the $\mathrm{Re}_{x}=2.9 \times 10^{5}$ case. The velocity profile is steeper in the near wall region and there is a velocity defect in the outer region. Both observations are consistent with turbulent boundary layer results. The rms velocity profile in Fig. 2(b) clearly shows that the $\operatorname{Re}_{x}=2.9 \times 10^{5}$ case boundary layer is not laminar as the fluctuation levels seen in the near wall region reach $14 \%$. These rms values are characteristic in level and position of those of a turbulent boundary layer. ${ }^{7,23}$ The uncertainty in the mean and rms velocity data were estimated to be $1.0 \%$ and $0.2 \%$, respectively.

The $\mathrm{Re}_{x}$ for the "naturally" turbulent (case 5) condition is somewhat low to be considered fully turbulent, and the absence of the wall at the measurement location may introduce further uncertainty in the rms velocity data. Thus, the raw velocity data were investigated further to better understand the nature of the inlet conditions. The data near the peak in the rms velocity data, however, showed no lowfrequency structure that would indicate intermittency or flapping of the half-jet, and the power spectra showed a nearequilibrium roll off. Thus, we do not believe the measurements to be biased by the measurement position. However, the nonstandard value of the shape factor when compared to fully developed turbulent boundary layers suggests that the boundary layer has only recently transitioned to turbulent flow. Given the intermediate value of the $\mathrm{Re}_{x}$ for case 5, it will be referred to herein as "turbulenttransitional" to avoid misrepresentation. Differences between this turbulent-transitional case and those with tripped high-speed boundary layers will be discussed below.

Hot wire anemometry measurements performed in the freestream showed that the turbulence intensity was less than
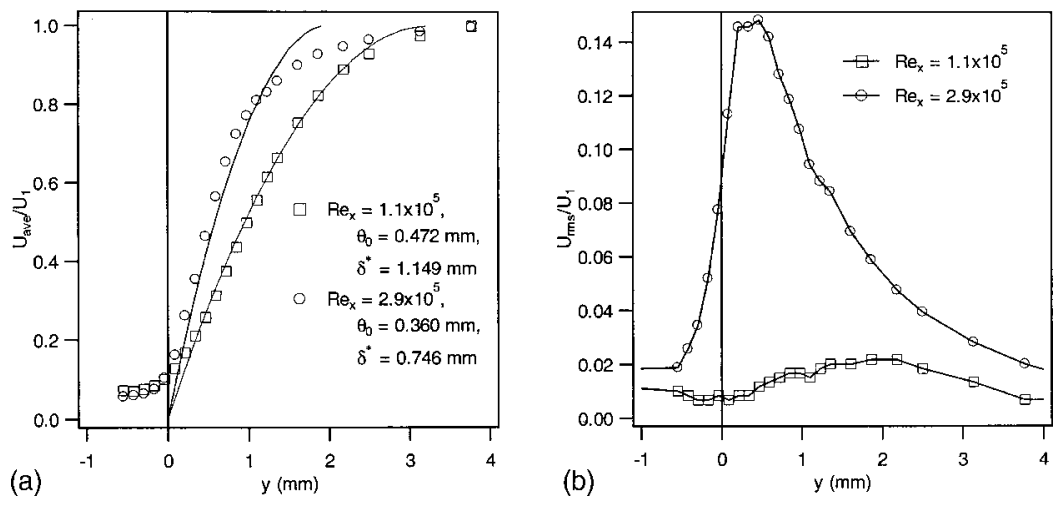

FIG. 2. Splitter plate high-speed boundary layer profiles acquired $1 \mathrm{~mm}$ past the splitter plate tip, (a) average and (b) rms velocity. The lower plate $\mathrm{Re}_{x}$ corresponds to the conditions of case 2 and the higher $\mathrm{Re}_{x}$, case 5. 
$0.6 \%$, and the velocity profile was uniform to within $1.3 \%$ across the span of the inlet for atmospheric conditions. Further, under the pressurized conditions of Table I, power spectra derived from hot wire measurements in the freestream indicated no clear acoustical modes or frequencies, and confirmed that the turbulence intensity was less than $0.6 \%$. Therefore, it can be stated with confidence that the effects of the inlet conditions arise from the boundary layer, and are not due to freestream disturbances.

The test cases listed in Table I are taken at $\operatorname{Re}_{\delta}$ that are considered transitional in nature. ${ }^{24}$ In our facility under laminar inlet conditions, transition at $\operatorname{Re}_{\delta}=5000$ was typical. ${ }^{20}$ This study, therefore, concentrates on effects at low and transitional $\operatorname{Re}_{\delta}$ where the effects of inlet conditions are more pronounced. But, as the study by Slessor et al. ${ }^{15}$ demonstrated, inlet conditions continue to be important at very high $\operatorname{Re}_{\delta}$. Another consequence of the conditions chosen is that the pairing parameter, $R x / \lambda_{0}$ is less than 5 for all cases. As will be discussed, the relevance of $R x / \lambda_{0}$ is less for cases with turbulent inlet conditions.

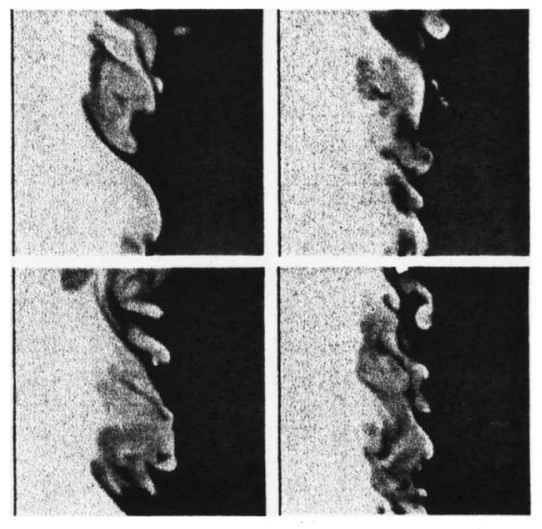

Case 1

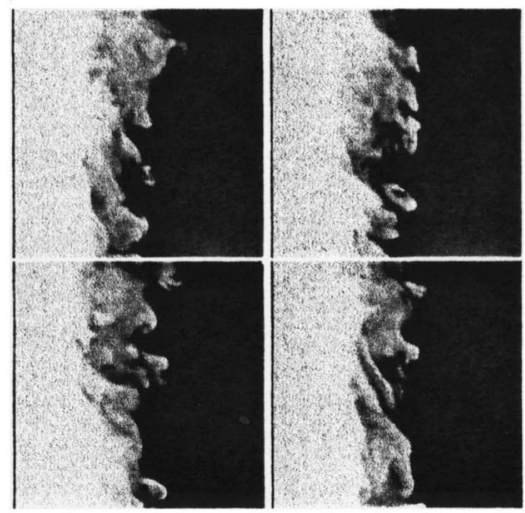

Case 3

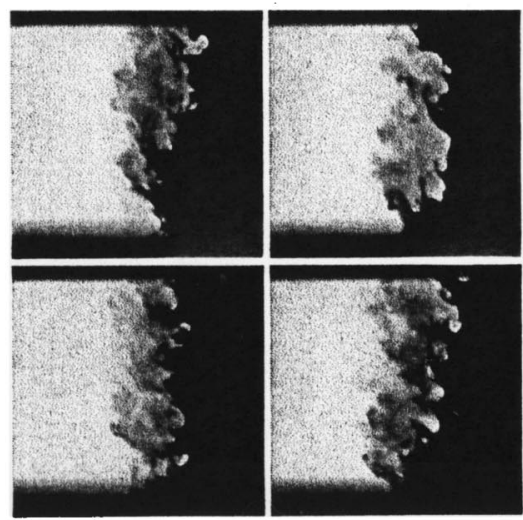

Case 5

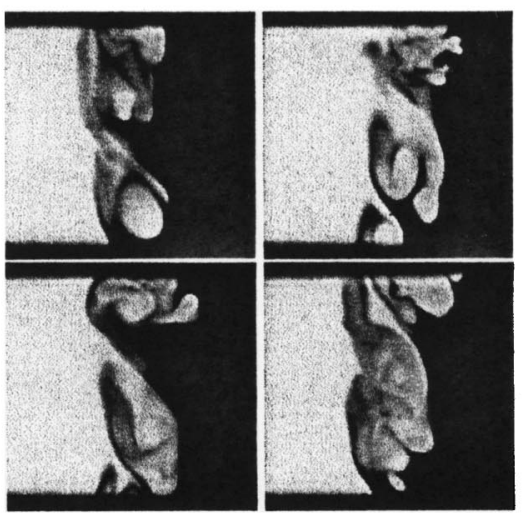

Case 2

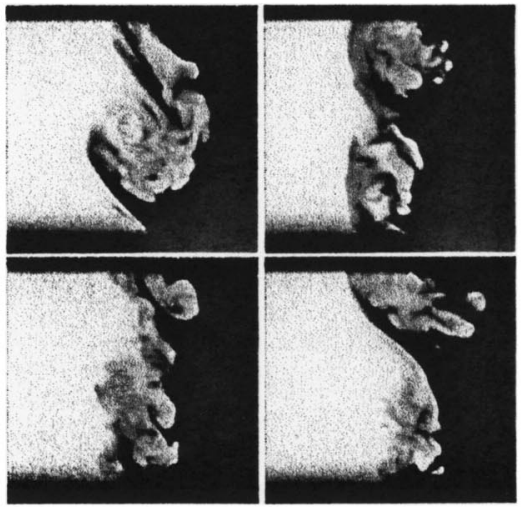

Case 4

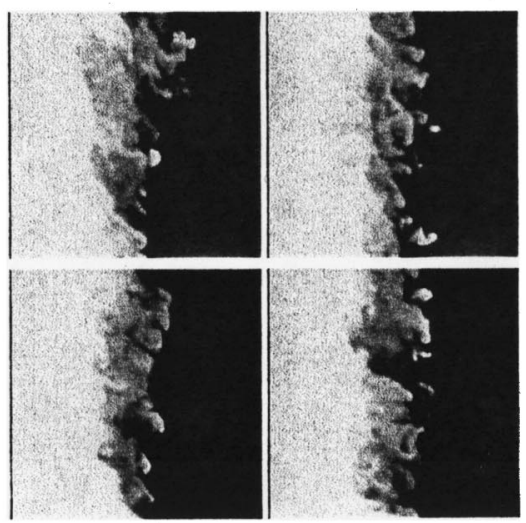

Case 6

FIG. 3. PLIF passive scalar images acquired at location B for operating conditions corresponding to cases 1-6. 


\section{RESULTS}

\section{A. Shear layer structure}

Four instantaneous PLIF realizations from image position B (see Fig. 1) for each of the cases 1-6 are shown in Fig. 3. The local $\operatorname{Re}_{\delta}$ at $x=11 \mathrm{~cm}$ for these cases are listed in Table I. The two cases with laminar inlet conditions (2 and 4) are characterized by large-scale structures of relatively homogenously mixed fluid which extend entirely across the mixing layer. Distinct core and braid regions are readily apparent with some evidence of structure pairing. Freestream fluid can be entrained entirely across the layer. At the lower $\operatorname{Re}_{\delta}$ of case 2 the layer is just beyond the turbulent transition, and the turbulent structures superimposed on the initial instability are relatively large. By $\operatorname{Re}_{\delta}=9600$, case 4 , the turbulent structures have broken down to scales significantly less than the layer width.

The effect of tripping the high-speed boundary layer with a nearly constant $\operatorname{Re}_{\delta}$ is seen by comparing cases 2 and 3 in Fig. 3. The promotion of a turbulent boundary layer prevents the rollup of the large-scale laminar KelvinHelmholtz structures. The mixing layer is instead dominated by small-scale turbulent structures which produce a relatively uniform gradient in the cross-stream direction, and freestream fluid does not entrain across the entire layer as it does with laminar inlet conditions. There is some visual evidence of large-scale structures but these structures are not separated by a distinct braid region. A similar set of observations are true when the high-speed boundary layer is turbulent-transitional (case 5). However, occasional images $(\sim 18 \%)$ showing identifiable large-scales structures separated by a distinct braid were found for the turbulent/ transitional boundary layer inlet condition. One such image is shown at the upper right in Fig. 3, case 5. No such occurrences were observed for either the strongly tripped laminar or tripped turbulent inlet conditions, cases 3 and 6, respectively.

The effect of weakly tripping the high-speed boundary layer, case 1, produces results which fall between the two limiting cases discussed previously. In spite of the fact that $\operatorname{Re}_{\delta} \approx 3000$ for this case, the layer has undergone turbulent transition and small-scale structures are evident. However, in several instances large-scale structures spanning the layer width are evident. In these cases freestream fluid is entrained across the layer, and mixing occurs in a fashion similar to post-transition conditions with laminar inlet boundary layers (case 4).

The differences observed in the shear layer structure can clearly be shown to result from the in-flow boundary layer conditions. Figure 4 shows images acquired closer to the splitter plate tip, position A in Fig. 1, for cases 2 and 5. The difference in the initial shear layer development with laminar and turbulent high-speed boundary layers is readily apparent.

The set of instantaneous images were analyzed to detect the presence of large-scale structures, especially for turbulent in-flow conditions. Following the technique used by Frieler, ${ }^{25}$ a cross-stream integrated instantaneous variance from the average mixture fraction, $\Xi(x)$, was defined at each streamwise position as
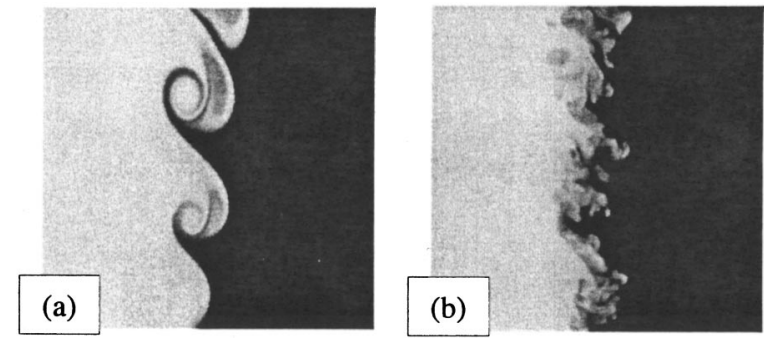

FIG. 4. PLIF images acquired at location A, (a) case 2, (b) case 5.

$$
\Xi(x)=\int_{-\infty}^{\infty}(\xi(x, y)-\bar{\xi}(x, y)) d y .
$$

This integral will be sensitive to passing large-scale structures that occupy the entire width of the mixing layer. An autocorrelation, $R(\ell)$, was then performed for each individual image as

$$
R(\ell)=\frac{\int \Xi(x) \Xi(x+\ell) d x}{\int \Xi^{2}(x) d x},
$$

where $\ell$ represents the separation distance. The individual results were summed over the entire image data set. The autocorrelation results are shown in Fig. 5 for cases 1-6 at image location $\mathrm{B}$, and for cases 2 and 5 at location $\mathrm{A}$.

The laminar, pre-transition condition of case $2 \mathrm{~A}$ provides an opportunity to assess this method for identifying structure spacing. The autocorrelation reaches a minimum at $\ell \mid \delta$ $=0.6$ and a maximum extrema at $\ell / \delta=1.2$. Dimensionally, this corresponds to a length of $1.4 \mathrm{~cm}$ which is in excellent agreement with Fig. 4. Furthermore this provides a Strouhal number, $S t=\theta_{0} / \ell=0.033$ based on the measured $\theta_{0}$ in excellent agreement with the theoretical value of $0.032 .{ }^{26}$ This excellent agreement between the autocorrelation results and measured structure spacing from the PLIF images provides confidence in the autocorrelation method for detecting largescale structures. The autocorrelation for the turbulent/ transitional inflow condition at position A (case 5A) also

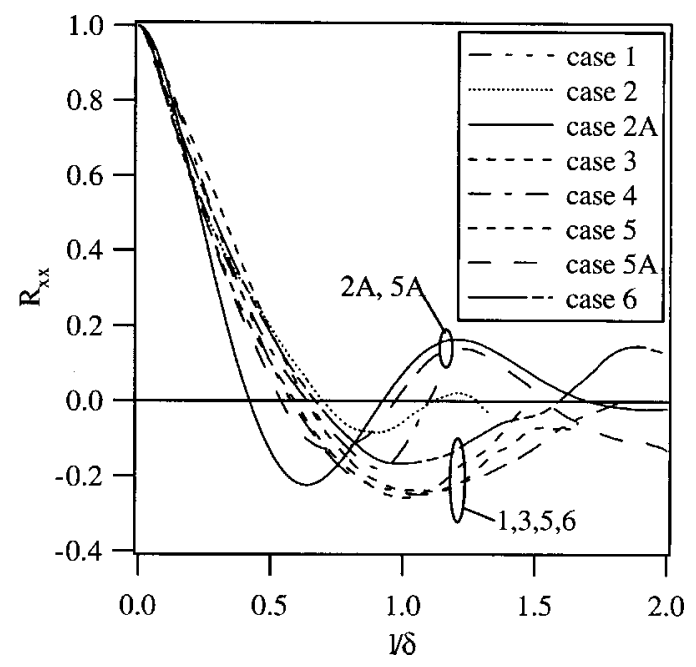

FIG. 5. Autocorrelation of integral mixture fraction variance. 

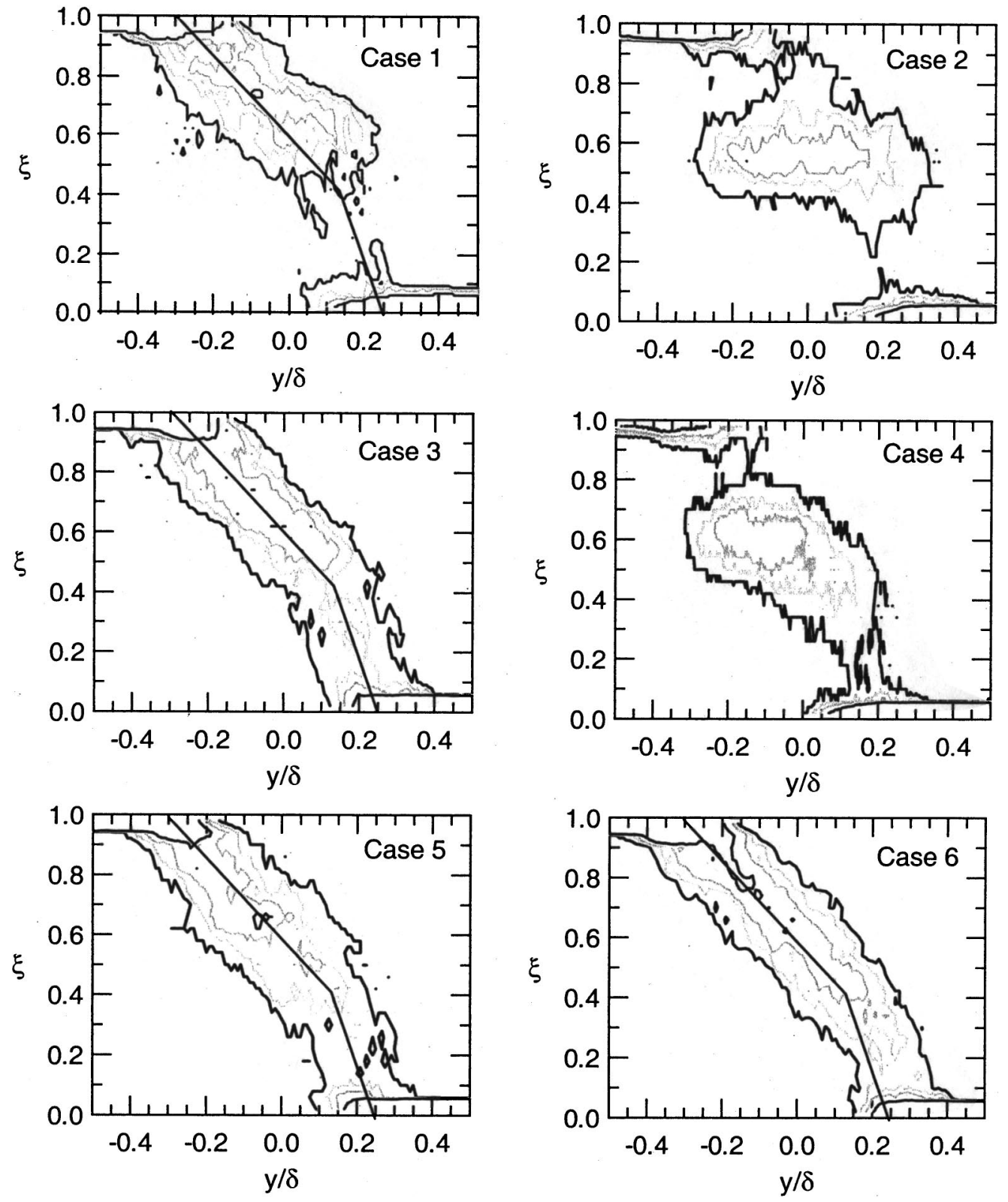

FIG. 6. Mixture fraction PDFs for cases $1-6$, computed at $x=11 \mathrm{~cm}$.

achieves a local maxima at $\ell / \delta=1.2$ despite a significant difference in the layer thickness from laminar inflow case. Unlike the laminar case, the local minima in the autocorrelation does not occur at half the distance to the maximum.

The autocorrelations performed at position B are limited by the image size. As such, the positive correlation corresponding to a full structure length is not observed, and the negative correlation corresponding to the half spacing will be used for discussion. It can be seen in Fig. 5 that all of the cases with turbulent inflow conditions exhibit a strong negative correlation at $\ell / \delta=1.1$ suggesting that large-scale structures are present in the layer despite the disorganized appearance in Fig. 3. The autocorrelation for the laminar inflow conditions at position B display a minima at a slightly lower $\ell \mid \delta$.

\section{B. Shear layer mixing}

Mixture fractions PDFs for cases $1-6$ at a streamwise position of $x=11 \mathrm{~cm}$ are shown in Fig. 6. The data have been normalized by the measured $1 \%$ thickness, and the $y$ $=0$ location is defined as the midpoint of the $1 \%$ and $99 \%$ mixture fraction positions. The PDFs are displayed as contour plots where lines at $p(\xi) \Delta \xi=0.04,0.06,0.08,0.10$, and 0.12 are drawn in each plot. The high-speed side $(\xi=1)$ is at the left at $y / \delta \leqslant-0.5$ while pure low-speed fluid $(\xi=0)$ is found at $y / \delta \geqslant 0.5$. The uncertainty in the PDFs is difficult to assess directly, but uncertainty in the derived quantities will be discussed fully below. Contour plots have been presented instead of three-dimensional (3D) projections to allow quantitative comparison independent of perspective. 
The PDFs for the two laminar inlet condition cases $(2$ and 4) exhibit a nonmarching behavior, as a preferred mixedfluid composition slightly greater than $\xi=0.5$ extends from one side of the mixing layer to the other. The nonmarching PDF style originates from the large, homogeneously mixed structures that typified the laminar inlet case in Fig. 3. The preferred mixed fluid composition is slightly higher in value for case 4 owing to the lower velocity ratio and higher entrainment rate of high-speed fluid. ${ }^{27}$ There is some evidence of a slight "tilt" in the PDF shape with higher $\operatorname{Re}_{\delta}$.

The effect of tripping the laminar boundary layer for $\operatorname{Re}_{\delta} \approx 5000$, case 3 , is quite significant. The PDF for case 3 exhibits a "hybrid" style, where the mixed fluid appears to march on the high-speed side, $y / \delta<0$, but displays a relatively flat, distributed style on the low-speed side, $y / \delta>0$. This distinct change in the PDF style from the laminar case is in agreement with the major differences observed in the instantaneous mixture fraction images, where tripping produced a mixing layer devoid of distinct large structures with clean braid regions or evidence of pairings.

The PDF for the turbulent-transitional inlet condition, case 5, also exhibits the hybrid shape, with a strong march on the high-speed side of the layer and a distributed structure on the low-speed side. (For comparison a solid line showing the hybrid PDF style has been added to all cases with turbulent inlet conditions in Fig. 6; where the shape was established based on case 3 and is unchanged between cases.) The PDF shows a slight widening near $\xi \approx 0.6$ (in terms of $y / \delta$, reflecting a slight preference in composition and less marching behavior) which is believed to be the effect of the occasional large-scale structure passing as was previously mentioned. The similarity of the PDF to that of case 3 is achieved in spite of the factor of 2 difference in $\operatorname{Re}_{\delta}$. Tripping the turbulent-transitional boundary layer, case 6 , causes only a slight change in the observed PDF style. A hybrid shape is retained, but a slightly more pronounced march is observed on the low-speed side of the layer. In addition, the PDF no longer exhibits the widening near $\xi \approx 0.6$, consistent with the lack of any large-scale structures observed in the instantaneous images. The small change observed upon tripping the turbulent-transitional case further supports the assertion that the boundary layer is past the transition state.

The weakly tripped inlet conditions again show a behavior which falls between that of the fully laminar or turbulent inlet condition limits. The PDF is seen to march on the highspeed side of the layer in Fig. 6, but not as strongly as the fully turbulent conditions and may be best described as having a tilted shape. The PDF exhibits a widening near $\xi$ $\approx 0.6$ again in accord with the existence of identifiable largescale structures. On the low-speed side of the mixing layer the PDF behavior is most reminiscent of the nonmarching laminar inlet conditions, where fluid either exists at a composition near $\xi \approx 0$ or $\xi \approx 0$.6. In all cases with perturbed inlet conditions it appears that the mixing processes differ from the high- to the low-speed side of the mixing layer.

Further details of the mixing process can be obtained from the PDF data using the derived quantities defined in Eqs. (2)-(4). For the turbulent inlet cases (3, 5, and 6) it was found that all of these derived data exhibited self-similar

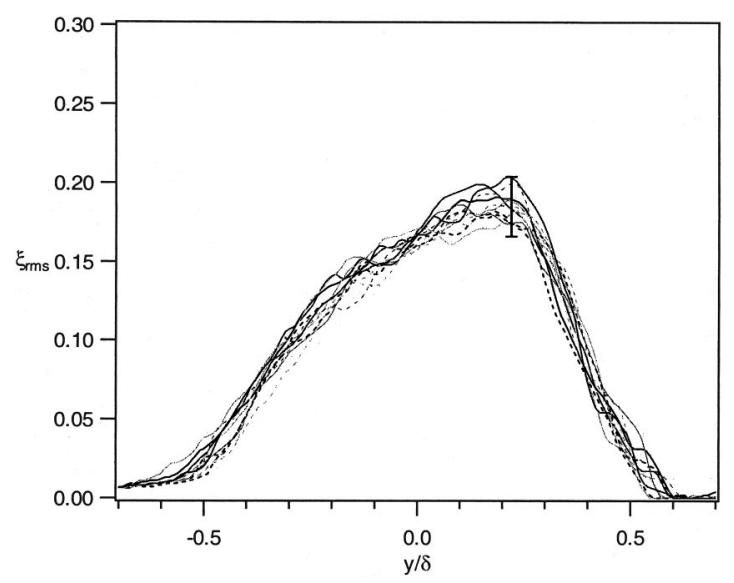

FIG. 7. Similarity of the rms mixture fraction profile for case 6 for distances ranging from 7.6 to $11.7 \mathrm{~cm}$ downstream of the splitter plate tip (7700 $\left.<\operatorname{Re}_{\delta}<11000\right)$.

behavior when scaled by the layer width $\delta$ over the spatial extent of Fig. 3. The rms mixture fraction profile is shown in Fig. 7 for case 6 at a range of downstream positions spanning the imaged area. These data span a range of $\mathrm{Re}_{\delta}$ from 770011000 , encompassing the $\operatorname{Re}_{\delta}$ of case 4 (laminar inlet conditions). It can be seen that self similarity has been achieved. The differences observed between the different curves can be used to (conservatively) estimate the uncertainty in the measurement technique. The uncertainty for each of the derived quantities $\xi_{\text {ave }}, \xi_{\text {rms }}, P_{m}$, and $\xi_{m}$ was defined as $\pm 2 \sigma$ where $\sigma$ was the maximum standard deviation measured in plots such as Fig. 7. In all subsequent plots this value will be shown attached to the case 3 data.

Figures 8(a)-(d) show the $\xi_{\text {ave }}, \xi_{\text {rms }}, P_{m}$, and $\xi_{m}$ profiles obtained at $x=11 \mathrm{~cm}$ for the turbulent inlet condition cases. There is excellent agreement between the three cases for all of the quantities. The relatively large uncertainty associated with the $P_{m}$ is considered to be an artifact of the definition of the layer width based on the $1 \%$ levels. The slight lateral shift in the data coupled with the high rate of change of the data produces unrealistically large uncertainty values. The value obtained at $y / \delta=0$ is shown as a reference and it is a factor of four less than the value defined by the procedure described above.

The mean mixture fraction profile has the classic shape with a gradual shift in composition across the layer with a maximum slope at $y / \delta \approx 0$. The RMS mixture fraction profile has an asymmetric shape, with a peak value occurring on the low-speed side of the layer, $y / \delta=0.2$. This is also the position where the hybrid PDF profile was found to transition from a marching to a distributed style. The mixed fluid probability $P_{m}$ peaks at the center of the mixing layer and symmetrically decreases on both sides. The peak value is close to 1.0 indicating that little freestream fluid is entrained across the center line. The mean mixed fluid mixture fraction $\xi_{m}$ was found to be strongly tilted, favoring the closer freestream value. The total mixed fluid mixture fraction calculated at $x=11 \mathrm{~cm}$ was the same for all the turbulent inlet conditions (not surprising given the coincidence of the $P_{m}$ 

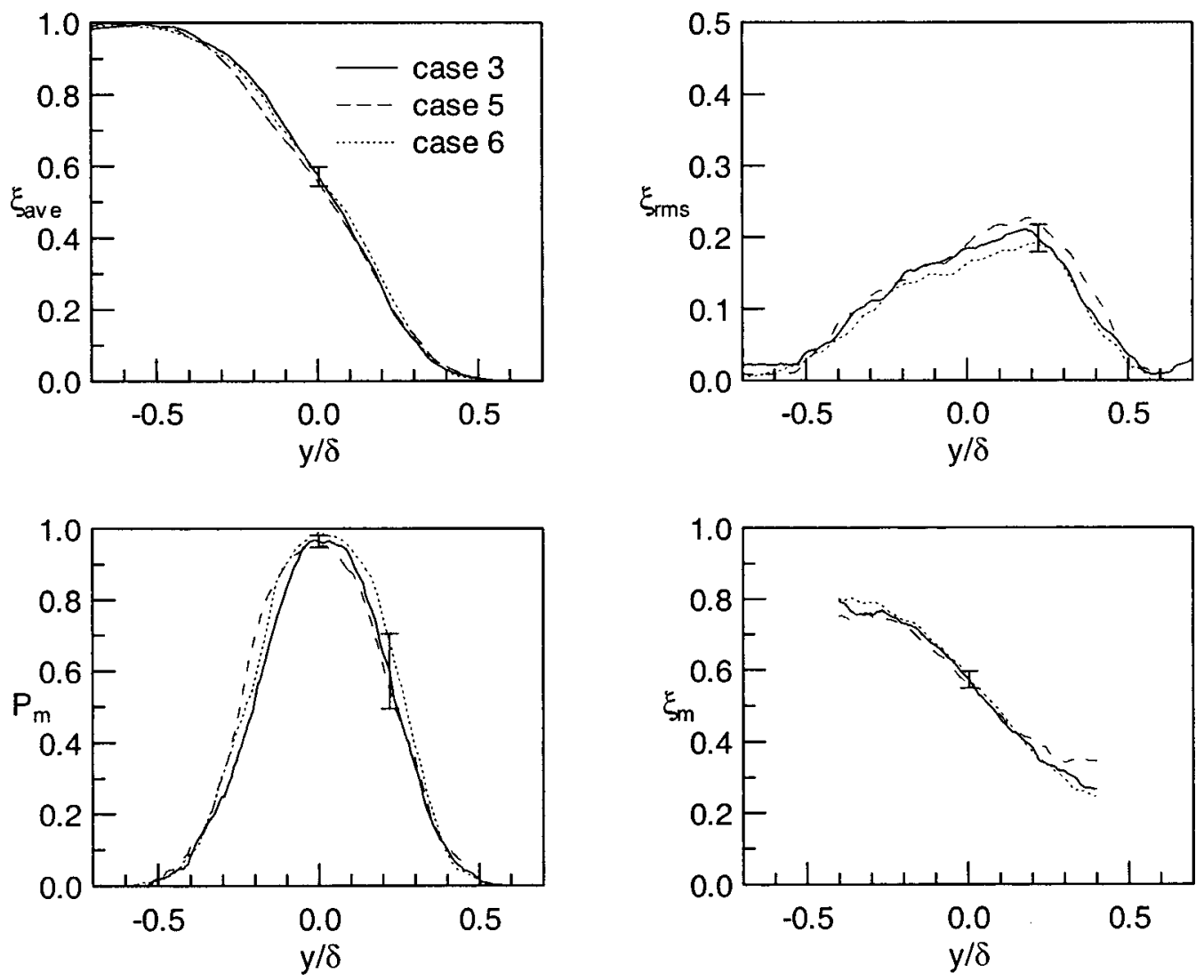

FIG. 8. Comparison of statistical quantities derived from the PDF data for cases with turbulent inlet conditions, case 3 (solid), case 5 (dashed), and case 6 (dotted).

and $\xi_{m}$ curves) $\xi_{M}=0.55$, and clearly shows a high-speed entrainment bias.

The self-similar behavior and qualitative agreement in the PDF shape between the turbulent inflow cases suggests that the turbulence is well developed. Hot-wire velocity data were obtained in the center of the mixing layer using the apparatus described previously, at a downstream position of $x=4.5 \mathrm{~cm}$. This position is approximately one-third of the distance from the bottom of the images in Fig. 4, and is well below the $x=11 \mathrm{~cm}$ position where the image data for statistical analysis were acquired. The power spectra of the velocity data are shown in Fig. 9. The tests were performed in a similar manner to the boundary layer data shown in Fig. 2, where the pressure was $1 \mathrm{~atm}$. The mass flow rates were the same as in cases 2 and $5\left(U_{1}=6 \mathrm{~m} / \mathrm{s}\right.$, case $2 ; U_{1}=16 \mathrm{~m} / \mathrm{s}$, case 5) therefore, the $\operatorname{Re}_{\delta}$ was approximately the same. Although the pressure was different from the actual tests, the structure spacing scales directly with the momentum thickness of the high-speed boundary layer which remained unchanged. ${ }^{27}$

The power spectra for case 2 where the boundary layer was laminar shows a peak corresponding to the KelvinHelmholtz rolloff frequency, $f=294 \mathrm{~Hz}$, which gives a Strouhal number, $S t=\theta_{0} f / \bar{U}=0.033$, in excellent agreement with predictions from linear stability theory ${ }^{27}$ and the previous length-scale measurements. The exponent of the power spectra for case 2 is near $n=-4$, indicating largely twodimensional behavior. For the turbulent inflow conditions of case 5 there were no clear peaks in the power spectra and the exponent is close to the equilibrium $-5 / 3$ value. The power spectra and PLIF images suggest that the turbulent inlet case has obtained a high degree of three-dimensional behavior and follows a standard energy cascade; and the flow has reached this state in a short streamwise distance past the splitter plate.

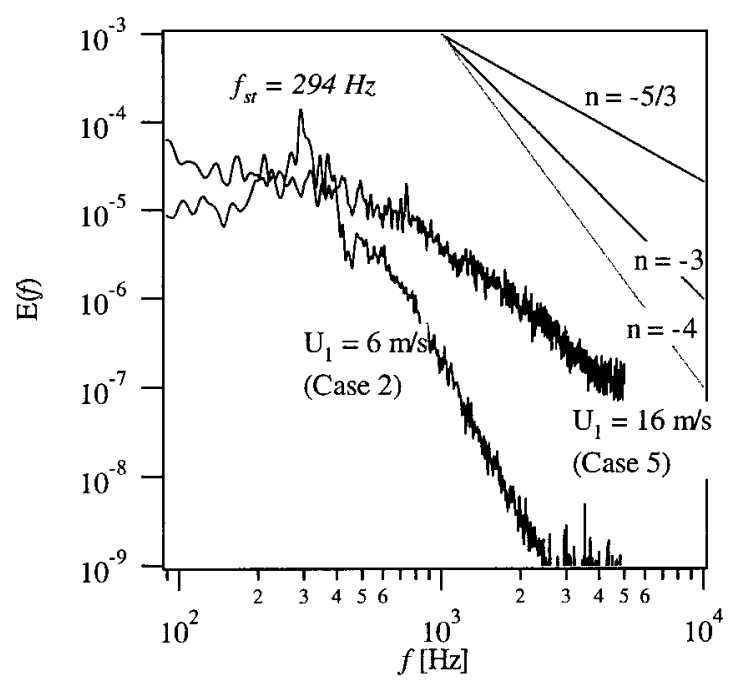

FIG. 9. Power spectra of velocity acquired at $x=4.5 \mathrm{~cm}$ and $y / \delta=0$. Data were acquired at atmospheric pressure with the same plate $\mathrm{Re}_{x}$ as case 2 $\left(U_{1}=6 \mathrm{~m} / \mathrm{s}\right)$ and case $5\left(U_{1}=16 \mathrm{~m} / \mathrm{s}\right)$. 

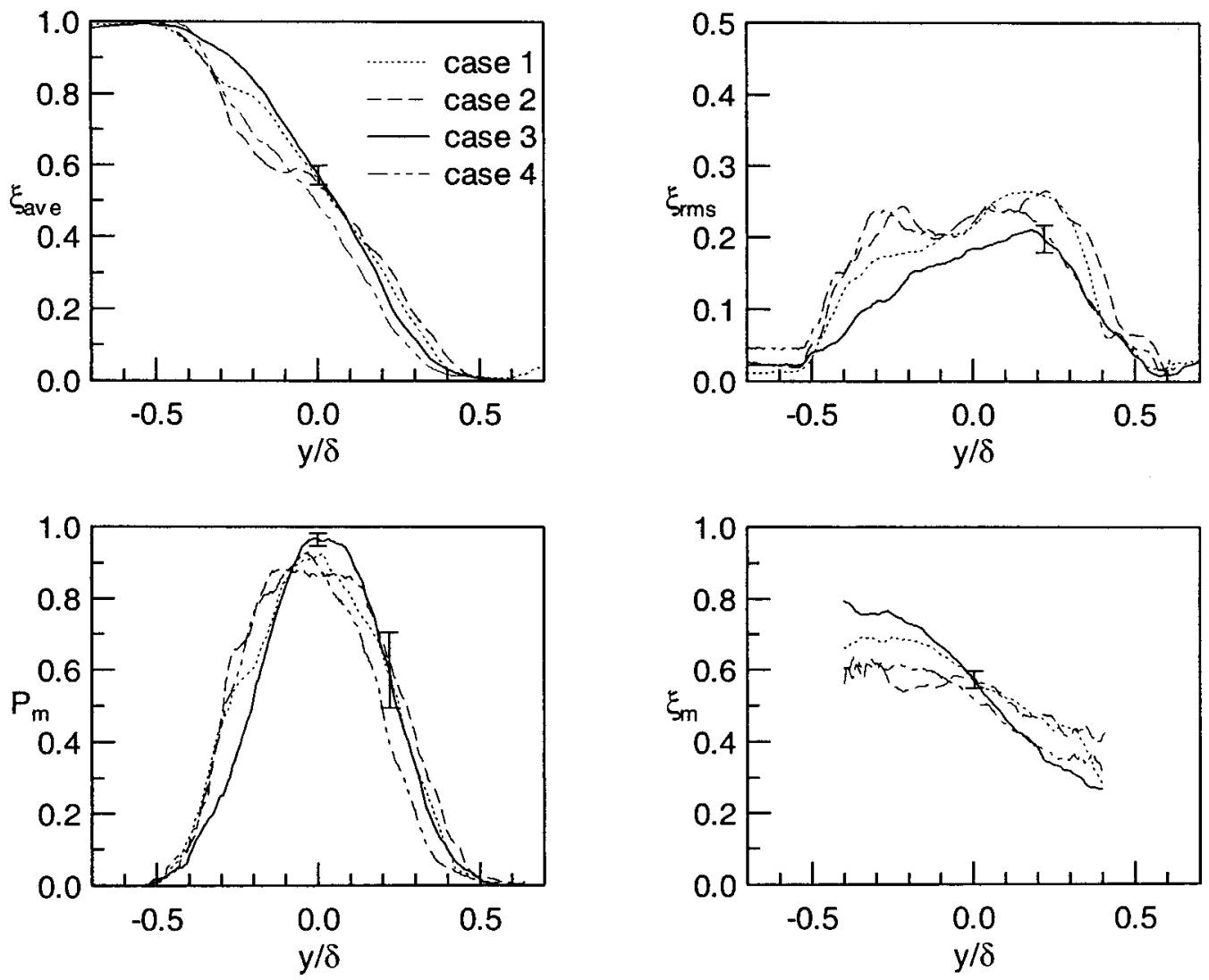

FIG. 10. Comparison of statistical quantities derived from the PDF data for cases with laminar inlet conditions, case 2 (dashed) and case 4 (dash-dot) with fully turbulent, case 3 (solid), and weakly tripped, case 1 (dotted), inlet conditions.

A comparison of the statistically derived quantities for the laminar (cases 2 and 4), weakly tripped (case 1) and turbulent (case 3) inlet conditions is shown in Figs. 10(a)$10(\mathrm{~d})$. The data were obtained at $x=11 \mathrm{~cm}$, and the uncertainty determined in the manner discussed previously is shown attached to the case 3 data.

Significant differences are observed for the different inlet conditions. The laminar and weakly tripped inlet conditions produce an average mixture fraction, $\xi_{\text {ave }}$, profile that has a triple inflection point. This is most clearly seen for case 2 where a plateau is observed at $\xi_{\text {ave }} \approx 0.6$ in Fig. 10(a). This behavior is the result of the nonmarching characteristic of the PDF that was found with laminar inlet conditions. The preferred composition of the nonmarching PDF manifests itself in the first moment of the distribution resulting in the plateau observed at this level. The mean mixed-fluid mixture fraction, $\xi_{m}$, shown in Fig. 10(d), also reflects this trend. The laminar inlet condition cases produce a relatively flat $\xi_{m}$ curve that is insensitive to location within the mixing layer, suggesting a single, preferred mixed fluid composition. In contrast, the turbulent inlet conditions produce a tilted $\xi_{m}$ profile that favors the nearest freestream value. The weakly tripped condition again exhibits an intermediate behavior, showing a more highly tilted structure on the high-speed side of the layer and a flatter profile on the low-speed side where the PDF was nonmarching.

The mixture fraction rms profiles differ significantly depending on the inlet conditions. For the laminar cases the profiles are nearly symmetric, and everywhere larger in magnitude than that of the turbulent inlet condition case. For the weakly tripped condition an asymmetric profile is observed with high values observed on the low-speed side of the mixing layer similar to the turbulent inlet condition case, but the rms magnitude is higher. The increase in magnitude of the rms mixture fraction in the laminar and weakly tripped cases are considered to be a result of the large-scale structures that were observed in Fig. 3. The passing of a large-scale structure produces a toggling of the mixture fraction from a freestream value to that of the preferred mixture composition of the structure, resulting in a high rms value.

\section{DISCUSSION}

\section{A. Comparison with previous studies}

Comparisons will be made with previous studies based on the inlet conditions. The laminar inlet condition results will be compared to low $\operatorname{Re}_{\delta}$ liquid and gaseous studies, although direct quantitative comparison is limited in part by the lower $\operatorname{Re}_{\delta}$ of these data. The turbulent inlet condition results exhibited self-similar behavior and a $-5 / 3$ slope in the power spectra, so they will be compared to fully developed conditions reported in the literature.

The nonmarching PDF observed with the laminar inlet condition cases compares quite favorably with the results of $\operatorname{Konrad}^{10}$ in a gaseous mixing layer, and the low $\mathrm{Re}_{\delta}$ results in liquid mixing layer studies. ${ }^{11,13}$ Further, the relatively in- 
variant $\xi_{m}$ across the layer width, and the triple inflection point behavior of the $\xi_{\text {ave }}$ profile are also consistent with previous findings. ${ }^{11,13}$ The peak value in the mixed fluid probability, $P_{m}$, is higher than observed in liquid phase studies due to the lower $S c$, and is not considered to be a resolution effect. ${ }^{20}$ Finally, the PLIF images clearly show the large-scale Kelvin-Helmholtz structures, and their persistence past the turbulent transition.

The turbulent inlet condition results are in good agreement with the resolution-free results of Frieler. ${ }^{25}$ The mean mixed-fluid mixture fraction tilts to favor the local freestream composition, although somewhat more highly in the present results. The peak in the mixed fluid probability at the center of the layer is in good quantitative agreement with Frieler's results, and further suggests that the resolution of the current passive scalar measurements is sufficient. In the liquid study of Karasso and Mungal ${ }^{13}$ the $P_{m}$ derived from their passive scalar measurements was shown to greatly exceed their resolution-free measurements.

In the present work the PDF shape was found to change very abruptly when the inlet conditions changed from laminar to turbulent. Karasso and Mungal ${ }^{13}$ showed a case below the critical value of the pairing parameter for which no shift from a nonmarching PDF style was observed with a highspeed trip $\left(\operatorname{Re}_{\delta}=14000\right)$. However, another sub-critical case in that study did toggle to a marching behavior with the addition of a trip on the high-speed side $\left(\operatorname{Re}_{\delta}=18500\right)$. In the present study, the pairing parameter for case 2 with laminar inlet conditions was 2.9 , much less than their critical value, yet tripping produced a significant change in the PDF shape (compare cases 2 and 3 in Fig. 6). Based on estimates from the data provided in Ref. 13 the tripping conditions were insufficient for their $\operatorname{Re}_{\delta}=14000$ case $\left(d_{\text {trip }} / \theta_{0}=5.7\right.$, $\mathrm{Re}_{\text {trip }}=630$, and $x_{\text {trip }} / \theta_{0}=-230$ ), and somewhat better, but not completely satisfied for their $\operatorname{Re}_{\delta}=18500$ case $\left(d_{\text {trip }} / \theta_{0}\right.$ $=8, \mathrm{Re}_{\text {trip }}=707$, and $x_{\text {trip }} / \theta_{0}=-300$ ). It was observed in the present results that inadequate tripping produces results intermediate to the limit cases, and this is believed to be the cause of the behavior observed in Ref. 13. The use of $R x / \lambda_{0}$ does not appear to be a relevant indicator for the turbulent inlet boundary layer conditions. Qualitatively, the observed change in the shear layer mixing upon tripping is in agreement with the results of Slessor et al. ${ }^{15}$

The hybrid PDF shape obtained with turbulent inlet conditions in the present work is considered to be unique. Karasso and Mungal ${ }^{13}$ found marching PDFs for cases with high $\operatorname{Re}_{\delta}$ or cases that were strongly tripped. However, their results were under-resolved, and they argue heuristically that in the asymptotic limit a tilted PDF profile results. Frieler ${ }^{25}$ inverted his time-temperature measurements to predict PDFs of the scalar field. On the low-speed side of the layer at his highest $\operatorname{Re}_{\delta}$ the results compare favorably with the hybrid PDF observed in this work. However, the calculated PDFs of Frieler $^{25}$ on the high-speed side of the layer differ significantly from the marching structure observed herein. In that work, there was more of a preferred composition that tilted slightly to the local freestream as compared to the strong march observed on the high-speed side of the layer in these results. In turbulent jet studies PDFs with both marching and non-marching components have been observed ${ }^{19,28}$ at high $\operatorname{Re}_{\delta}$ conditions. However, in those studies the PDF was found to march on the low-speed side rather than the highspeed side as in this study; and the nonmarching behavior of the high-speed side differs from the broadening of the PDF observed in this work. Further discussion of the PDF shape and its implications are provided in the following section.

\section{B. Mixing imbalance}

The hybrid PDF shape suggests that there is a difference in how mixing occurs between the high- and low-speed sides of the mixing layer. On the high-speed side of the layer the marching PDF shape suggests a gradient diffusion-like entrainment process. On the low-speed side of the layer, the relatively flat PDF shape merely suggests a bias of low-speed fluid. In spite of the observed asymmetry of the PDF, no significant asymmetries in the mean mixture fraction, mixed fluid probability and the mean mixed-fluid mixture fraction are observed.

In all of the cases for which the hybrid PDF shape was observed the high-speed boundary layer was turbulent or transitional, but the low-speed boundary layer was laminar. To investigate whether the observed mixing imbalance was the result of the different states of the splitter plate boundary layers, a test was performed with a low-speed boundary layer trip for a case where the high-speed boundary layer was turbulent-transitional, case 8 in Table I. The conditions of the low-speed boundary layer for case 8 are nearly identical to those of the high-speed boundary layer of case 3 , so we are confident that we are in the strongly tripped limit.

A comparison of the statistical properties derived from the PDF for the tripped low-speed boundary layer, case 8, and that of the laminar low-speed boundary layer, case 5, is shown in Fig. 11. Excellent agreement is seen between these two cases, with the small discrepancies much less than the uncertainties discussed in Figs. 7 and 8. These two data sets were acquired more than one calendar year apart, providing further confidence in the experimental apparatus. The PDF for case 8 is not shown, but exhibited the hybrid shape and agreed well with the case 5 PDF.

The lack of an effect of a low-speed boundary layer trip is consistent with previous investigators. ${ }^{15}$ More importantly, the lack of an effect from the low-speed trip indicates that the mixing imbalance cannot be explained by inlet conditions. Thus, the mixing imbalance is the result of the free shear layer development when the high-speed boundary layer is turbulent. It should be noted that the hybrid style of PDF is inconsistent with the Broadwell-Briedenthal model $^{29}$ for shear layer entrainment and mixing, which was based on a nonmarching style PDF.

A consistent, physical cause for the hybrid shaped PDF is not clear at this time, but several factors that may be of significance are considered here. DNS work by Cortesi et al. ${ }^{16}$ suggests that the tendency of the PDF to tilt may be caused by a balance between large-scale spanwise structures and cross-stream mixing promoted by smaller-scale, threedimensional stirring. The existence of large-scale structures in the mixing layer even when the boundary layer is turbu- 

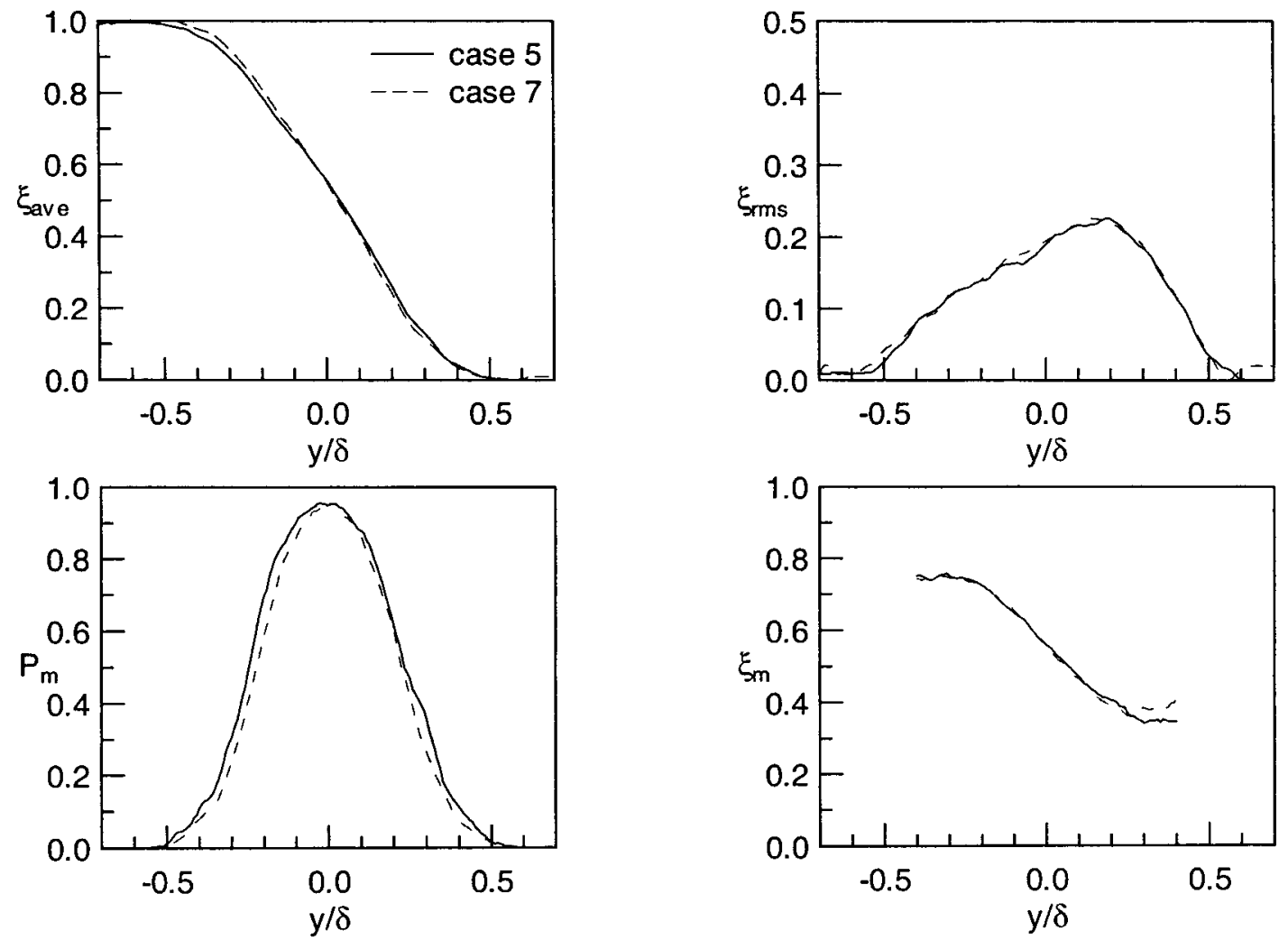

FIG. 11. Effect of low-speed trip for a condition with a turbulent-transitional high-speed boundary layer. Case 5 (solid) has no low-speed trip, Case 7 (dashed) has low-speed trip.

lent is consistent with the autocorrelation results presented in Fig. 5. The hybrid shape of the PDF could, therefore, be influenced by the interaction of the large and small-scale structures. However, the tilt or fully marching style PDF observed in temporally developed DNS studies is generally symmetrical about the center of the mixing layer. Temporally developed DNS studies are incapable of showing an entrainment bias of high-speed fluid which occurs in a spatially developed mixing layer ${ }^{26}$ (experimental mixing layers are spatially developed). Thus, the high-speed side entrainment bias may play a role in the hybrid shape of the PDF observed in the experiment.

In the recent literature a significant effort has been put forth to identify the asymptotic far-field PDF shape associated with shear layer development in planar ${ }^{10-13}$ and axisymmetric $^{19,28}$ configurations. As shown by Slessor et al., ${ }^{15}$ and confirmed in part by this work, there does not appear to be a unique shape that is independent of inlet conditions. However, it is interesting to note that in these results all conditions with a turbulent high-speed boundary layer produced the same hybrid PDF shape; and the quantities derived from the passive scalar PDF had achieved selfsimilarity. Further, the hybrid shape was observed for turbulent inlet conditions obtained in several different ways (with turbulent-transitional, tripped laminar and tripped turbulent-transitional high-speed splitter plate boundary layers). Lastly, the power spectra obtained from hot wire anemometry measurements exhibited a $-5 / 3$ exponent. Thus, by all conventional metrics the flow field obtained with tur- bulent high-speed inlet conditions is fully developed at this location.

\section{Secondary structures}

A unique, secondary Kelvin-Helmholtz rollup was observed in some images for cases with turbulent-transitional inlet boundary conditions, cases 5 and 7. Shown in Fig. 12 are sample images taken from image location B for these cases. Large-scale structures are clearly evident in both cases. In the braid region, a chain of spanwise vortices are observed suggesting that a localized shear layer has formed.

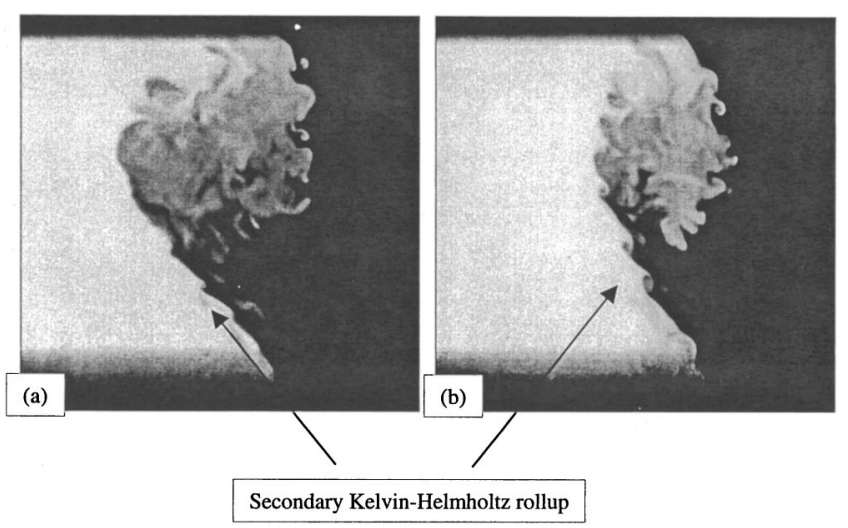

FIG. 12. Secondary structures observed at image position B (a) case 5, (b) case 7. 
As this shear layer is not connected directly to the original Kelvin-Helmholtz instability it is termed a secondary rollup.

Secondary rollups were not observed in every image, but did occur in approximately $5 \%$ of the images at these flow conditions. Additionally, secondary rollups were not observed for any case where the inlet conditions were laminar, or where the laminar boundary layer was tripped. The definition of what constitutes a secondary rollup is somewhat arbitrary from simple flow visualization as many smallstructure fingerlings are frequently seen, however, in no cases other than 5 or 7 were the structures so organized and clear.

Moser and Rogers ${ }^{30}$ observed similar structures in a DNS study, but no previous experimental reporting of such secondary structures was found in the literature. The threedimensional simulation of Moser and Rogers extended through the turbulent transition, and several inlet boundary conditions were simulated. When the inlet boundary condition included three-dimensional disturbances with high streamwise vorticity the secondary structures were observed. The structures were shown to occur at the stagnation plane between two counter-rotating streamwise vortices. Under this condition, a localized step-change in streamwise velocity could be maintained in the cross-stream direction, and created a localized shear layer and subsequent laminar KelvinHelmholtz rollup. Unlike the original rollup, the secondary rollup is a localized event and does not span the entire test section. This localized behavior could be the reason for the relatively infrequent observation of the secondary structure in the experimental data.

Although there is no way to directly prove that the structures are caused by the process described in the study of Rogers and Moser, the similarity between the necessary inlet conditions for their existence is striking. Development of streamwise vorticity in the boundary layer is characteristic of transitional boundary layers, ${ }^{23}$ and streamwise vorticity in the inlet conditions was necessary to observe the secondary structures in the DNS study. ${ }^{30}$ As noted above, the tripped cases did not support the secondary rollup. This subtle change in the inlet condition between a turbulenttransitional layer and a forced turbulent layer may be responsible for this difference.

\section{CONCLUSIONS}

Passive scalar measurements were performed in a twostream planar mixing layer to investigate the effect of inlet conditions on the mixing process. High-speed boundary layers that were laminar, turbulent-transitional and tripped, and low-speed boundary layers that were laminar and tripped were tested. The resolution of the acetone PLIF measurements was near the mass diffusion length scale $\left(1.2<L / \lambda_{D}\right.$ $<3.2$ ), so it is expected that there was minimal subresolution mixing, and the measurements were adequately resolved.

With laminar inlet conditions the layer was found to develop in a manner consistent with previous studies. The initial Kelvin-Helmholtz instability grew into large-scale struc- tures that persisted past the turbulent transition $\left(\operatorname{Re}_{\delta} \approx 5000\right.$ in this apparatus). The measured mixture fraction PDF showed a non-marching style with a high-speed entrainment bias. The mean mixed-fluid mixture fraction profile was nearly constant across the mixing layer, and the rms mixture fraction was nearly symmetric.

Tripping the laminar high-speed splitter plate boundary layer significantly changed the PDF shape from the laminar case at a similar $\operatorname{Re}_{\delta}$ (cases 2 and 3 in Fig. 6). The resultant PDF had a hybrid structure that was characterized by a marching shape on the high-speed side of the layer that transitioned to a relatively flat, broad shape on the low-speed side of the mixing layer. The existence of a turbulenttransitional high-speed stream boundary layer also produced a significantly different PDF shape compared to the laminar high-speed boundary layer case at a similar $\operatorname{Re}_{\delta}$ (cases 4 and 5 in Fig. 6). The PDF for the turbulent-transitional highspeed boundary layer exhibited the same hybrid shape as the tripped laminar case. Tripping the turbulent-transitional case did not alter the hybrid PDF shape. Thus, for all turbulent high-speed inlet conditions a hybrid-style PDF was obtained independent of $\operatorname{Re}_{\delta}$, and is a unique finding of this study. The statistical quantities derived from the PDFs were identical within the accuracy of the measurements for all of the turbulent high-speed boundary layer cases. Further, all turbulent profiles were found to be self-similar when scaled by the layer width, and the power spectra from (limited) hot-wire anemometry measurements exhibited a $-5 / 3$ slope, suggesting that a well developed turbulent state had been established a short distance from the splitter plate tip and at low values of $\operatorname{Re}_{\delta}$ and the pairing parameter.

The asymmetrical shape of the hybrid PDF suggests that the mixing process differs between the high- and low-speed sides of the mixing layer. The presence of a low-speed boundary layer trip when the high-speed boundary layer was turbulent-transitional produced no discernable difference in the PDF shape, or the statistical quantities derived from it. This confirmed that the asymmetrical hybrid PDF shape is the result of the natural evolution of the mixing layer with a turbulent high-speed inlet condition, not the result of a difference between the inlet conditions of the two streams. The passive scalar results were found to be sensitive to the level of perturbation introduced by the trip wire, with the existing criteria in the literature accurately reflecting the necessary conditions.

Finally, the naturally turbulent high-speed boundary layer cases gave rise to the occasional appearance of secondary structures within the shear layer. These structures were not observed with tripped high-speed conditions. This difference between cases with nominally similar inlet conditions suggests that complete inlet flow characterization may not be possible, as even subtle differences of in-flow conditions affect the nature of the flow development.

\section{ACKNOWLEDGMENT}

Support for this work was provided by the Army Research Office under Grant No. DAAH04-94-G-0328. 
${ }^{1}$ R. G. Batt, "Some measurements on the effect of tripping the twodimensional shear layer," AIAA J. 13, 245 (1975).

${ }^{2}$ I. Wygnanski and H. E. Fiedler, "The two-dimensional mixing region," J. Fluid Mech. 41, 327 (1970).

${ }^{3}$ F. K. Browand and B. O. Latigo, "Growth of the two-dimensional mixing layer from a turbulent and non-turbulent boundary layer," Phys. Fluids 22, 1011 (1979).

${ }^{4}$ M. G. Mungal, J. C. Hermanson, and P. E. Dimotakis, "Reynolds number effects on mixing and combustion in a reacting shear layer," AIAA J. 23, 1418 (1985).

${ }^{5}$ J. H. Bell and R. D. Mehta, "Development of a two-stream mixing layer from tripped and untripped boundary layers," AIAA J. 28, 2034 (1990).

${ }^{6} \mathrm{P}$. Bradshaw, "The effect of initial conditions on the development of a free shear layer," J. Fluid Mech. 26, 225 (1966).

${ }^{7}$ A. K. M. F. Hussain and M. F. Zedan, "Effects of initial condition on the axisymmetric free shear layer: Effects of the initial momentum thickness," Phys. Fluids 21, 1100 (1978).

${ }^{8}$ P. E. Dimotakis and G. L. Brown, "The mixing layer at high Reynolds number: Large-structure dynamics and entrainment," J. Fluid Mech. 78, 535 (1976)

${ }^{9}$ L. S. Huang and C. M. Ho, "Small-scale transition in a plane mixing layer," J. Fluid Mech. 210, 475 (1990).

${ }^{10}$ J. H. Konrad, Ph.D. thesis, California Institute of Technology, 1976.

${ }^{11}$ M. M. Koochesfahani and P. E. Dimotakis, "Mixing and chemical reactions in a liquid mixing layer," J. Fluid Mech. 170, 83 (1986).

${ }^{12}$ R. G. Batt, "Turbulent mixing of passive and chemically reacting species in a low-speed shear layer," J. Fluid Mech. 82, 499 (1977).

${ }^{13}$ P. S. Karasso and M. G. Mungal, "Scalar mixing and reaction in plane liquid shear layers," J. Fluid Mech. 323, 23 (1996).

${ }^{14}$ K. A. Buch and W. J. A. Dahm, "Experimental study of the fine-scale structure of conserved scalar mixing in turbulent shear flows," J. Fluid Mech. 364, 1 (1998).

${ }^{15}$ M. D. Slessor, C. L. Bond, and P. E. Dimotakis, "Turbulent shear-layer mixing at high Reynolds numbers: Effects of inflow conditions," J. Fluid Mech. 376, 115 (1998).
${ }^{16}$ A. B. Cortesi, B. L. Smith, B. Sigg, and S. Banerjee, "Numerical investigation of the scalar probability density function distribution in neutral and stably stratified mixing layers," Phys. Fluids 13, 927 (2001).

${ }^{17}$ M. M. Rogers and R. D. Moser, "Direct simulation of a self-similar turbulent mixing layer," Phys. Fluids 6, 903 (1994).

${ }^{18}$ A. Ansari, "Self-similarity and mixing characteristics of turbulent mixing layers starting from laminar initial conditions," Phys. Fluids 9, 1714 (1997).

${ }^{19}$ G. F. King, J. C. Dutton, and R. P. Lucht, "Instantaneous, quantitative measurements of molecular mixing in the axisymmetric jet near field," Phys. Fluids 11, 403 (1999).

${ }^{20}$ L. M. Pickett and J. B. Ghandhi, "Passive scalar measurements in a planar mixing layer by PLIF of acetone," Exp. Fluids 31, 309 (2001).

${ }^{21}$ L. M. Pickett, Ph.D. dissertation, University of Wisconsin-Madison, 2000.

${ }^{22}$ A. Lozano, B. Yip, and R. K. Hanson, "Acetone: A tracer for concentration measurements in gaseous flows by planar laser-induced fluorescence," Exp. Fluids 13, 369 (1992).

${ }^{23}$ J. O. Hinze, Turbulence (McGraw Hill, New York, 1975).

${ }^{24}$ P. E. Dimotakis, "Turbulent free shear layer mixing with fast chemical reactions," in Turbulent Reacting Flows, edited by R. Borghi and N. B. Murthy, Lectures Notes in Engineering (Springer, New York, 1989), Vol. 40, p. 417.

${ }^{25}$ C. E. Frieler, Ph.D. thesis, California Institute of Technology, 1992.

${ }^{26}$ C. M. Ho and P. Huerre, "Perturbed free shear layers." Annu. Rev. Phys. Chem. 16, 365 (1984).

${ }^{27}$ P. E. Dimotakis, "Two-dimensional shear layer entrainment," AIAA J. 24, 1791 (1986).

${ }^{28}$ N. T. Clemens and P. H. Paul, "Scalar measurements in compressible axisymmetric mixing layers," Phys. Fluids 7, 1071 (1995).

${ }^{29}$ J. E. Broadwell and R. E. Briedenthal, "A simple model of mixing and chemical reactions in a turbulent shear layer," J. Fluid Mech. 125, 397 (1982).

${ }^{30}$ R. D. Moser and M. M. Rogers, "The three-dimensional evolution of a plane mixing layer: Pairing and transition to turbulence," J. Fluid Mech. 247, 275 (1993). 